\title{
Four-terminal magnetoresistance of a two-dimensional electron-gas constriction in the ballistic regime
}

\author{
H. van Houten, C. W. J. Beenakker, and P. H. M. van Loosdrecht \\ Philips Research Laboratories, $5600 \mathrm{JA}$ Eindhoven, The Netherlands \\ T. J. Thornton, H. Ahmed, and M. Pepper \\ Cavendish Laboratory, Cambridge CB3 OHE, United Kingdom \\ C. T. Foxon and J. J. Harris \\ Philips Research Laboratories, Redhill, United Kingdom \\ (Received 22 February 1988)
}

\begin{abstract}
A novel negative magnetoresistance effect is found in four-terminal measurements of the voltage drop across a short constriction of variable width in a high-mobility two-dimensional electron gas. The effect is interpreted as the suppression by a magnetic field of the geometrical constriction resistance in the ballistic regime. Quantitative agreement with a simple model based on a Landauer-type formula is obtained.
\end{abstract}

In a two-dimensional electron gas (2D EG) the ballistic transport regime has become accessible because of advances in microstructure fabrication and the growth of high-mobility GaAs- $\mathrm{Al}_{x} \mathrm{Ga}_{1-x}$ As heterostructures. ${ }^{1}$ Injection of ballistic electrons in a $2 \mathrm{D} \mathrm{EG}$, and the existence of skipping orbits at the 2D EG boundary, was recently directly demonstrated in a transverse electron focusing experiment, ${ }^{2}$ while van Wees et al. ${ }^{3}$ and Wharam et al. ${ }^{4}$ reported the discovery of the quantized two-terminal resistance of ballistic point contacts. For a ballistic 2D EG in the absence of a magnetic field, one finds ${ }^{3}$ for the classical two-terminal resistance $R_{2 t}$

$$
R_{2 t}=\left(h / 2 e^{2}\right) \pi / k_{F} W,
$$

with $k_{F}=\left(2 \pi n_{s}\right)^{1 / 2}$ the Fermi wave vector and $n_{s}$ the electron concentration in the constriction of width $W$. The two-terminal resistance does not contain information on the distribution of the voltage drop over the sample. This distribution is the focus of interest in this paper.

Voltage probes on the sides of a narrow channel are a source of diffuse scattering. We have, therefore, chosen for an alternative geometry ${ }^{5}$ in which the voltage probes are positioned on wide $2 \mathrm{D}$ EG regions, adjacent to a smooth constriction consisting of a narrow channel of length $3.4 \mu \mathrm{m}$ and width between 0 and $1 \mu \mathrm{m}$ (see inset of Fig. 1). The sample is fabricated on a high-mobility GaAs- $\mathrm{Al}_{x} \mathrm{Ga}_{1}{ }_{x} \mathrm{As}$ heterostructure, with a sheet carrier concentration $n_{s}=3.25 \times 10^{15} \mathrm{~m}^{-2}$ and a mean free path $l=10 \mu \mathrm{m}$. By employing a split-gate lateral depletion technique ${ }^{6}$ to define the narrow 2D EG region, the channel width can be varied continuously. We observe a temperature-independent (between $50 \mathrm{mK}$ and $4 \mathrm{~K}$ ) negative four-terminal magnetoresistance $R_{4 t}$ once the narrow region is defined (see Fig. 1).

The result (1) for the two-terminal resistance in zero field corresponds to the classical limit of the quantummechanical expression ${ }^{3,7}$

$$
R_{2 t}=\left(h / 2 e^{2}\right) N_{\min }^{-1},
$$

with $N_{\min }$ the number of occupied 1D electric subbands in the constriction, where the 2D EG has its minimum width (for a square-well lateral confinement potential of width $W, N_{\min }$ is given by the largest integer smaller than $\left.k_{F} W / \pi\right)$. As demonstrated experimentally in Ref. 8, the two-terminal resistance of a ballistic sample in a magnetic field is still given by Eq. (2), but with $N_{\min }$ replaced by $N_{\min }(B)$, the number of occupied hybrid magnetoelectric subbands (or quantum channels) in the narrow region.

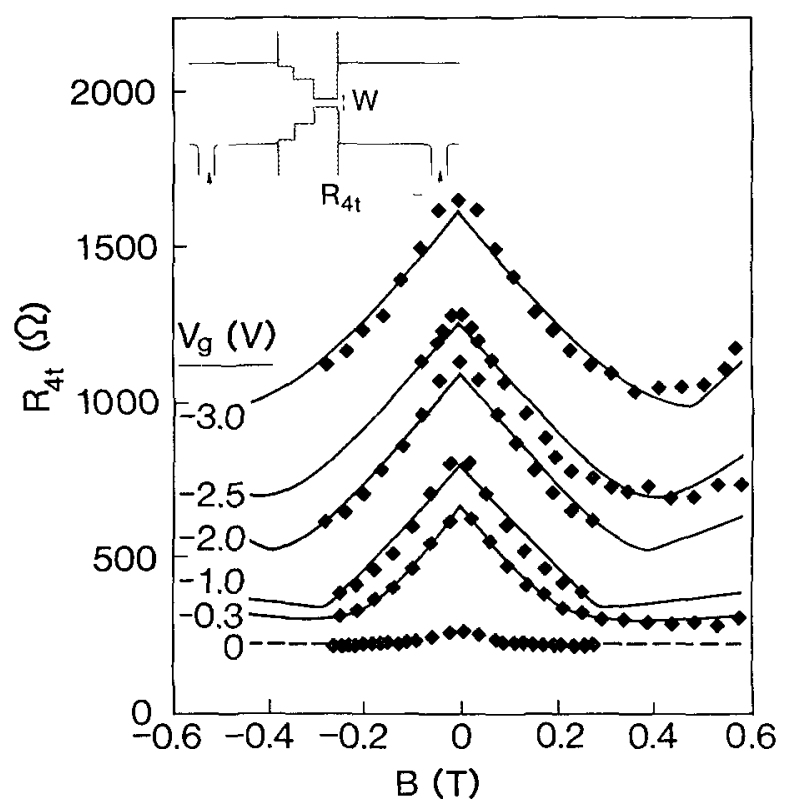

FIG. 1. Four-terminal magnetoresistance for various gate voltages. Solid lines are according to Eq. (4). The sample geometry is schematically shown in the inset (dashed lines indicate the depletion boundaries). The parameter values for the curves corresponding to gate voltages from $V_{g}=-0.3$ to -3.0 $\mathrm{V}$ are, respectively, $W=0.81,0.60,0.42,0.36$, and $0.29 \mu \mathrm{m}$ and $n_{s}=3.25,3.08,2.50,2.25$, and $1.90 \times 10^{15} \mathrm{~m}^{-2}$. 
Theoretically, this follows from the Landauer formalism ${ }^{7}$ by calculating the current carried in each subband by electrons injected into the constriction within a narrow range $\Delta \mu$ above the Fermi energy $E_{F}$. If the dispersion of the $n$th subband is given by the function $\epsilon_{n}(k)$, then one may write for the group velocity $v_{n}=\partial \epsilon_{n} / \hbar \partial k$ and for the density of (right-going) states $\rho_{n}=\left(\pi \partial \epsilon_{n} / \partial k\right)^{-1}$ (both evaluated at the Fermi level). Each subband thus carries the same current $e v_{n} \rho_{n} \Delta \mu=(2 e / h) \Delta \mu$. The total current $I=(2 e / h) \Delta \mu N_{\min }(B)$ yields a two-terminal resistance $R_{2 t} \equiv \Delta \mu / e I$ of the form (2). Assuming spin degeneracy, and ignoring the discreteness of $N$, one finds ${ }^{8}$

$$
\begin{aligned}
& N_{\min }(B)=\left(k_{F} l_{c} / \pi\right)\left\{\arcsin \left(W / 2 l_{c}\right)+\left(W / 2 l_{c}\right)\right. \\
&\left.\times\left[1-\left(W / 2 l_{c}\right)^{2}\right]^{1 / 2}\right\}, \text { if } W<2 l_{c},
\end{aligned}
$$

$N_{\min }(B)=k_{F} l_{c} / 2$, if $W>2 l_{c}$.

Here $l_{c} \equiv \hbar k_{F} / e B$ is the classical cyclotron radius. Equation (3) describes the crossover from electric subbands to Landau levels, as the magnetic field is increased.

To obtain an expression for $R_{4 t}$ from Eqs. (2) and (3), we consider the four-terminal geometry of Fig. 2. Electrons are injected at the left-hand side of the constriction, within $\Delta \mu$ above $E_{F}$. A fraction of these is transmitted through the constriction, leading to a current $I=\Delta \mu / e R_{2 t}$. In a perpendicular magnetic field, the incoming and transmitted electrons are localized at the upper boundary of the 2D EG in edge states at the Fermi level ${ }^{7}$ - provided the width of the regions leading to the constriction exceeds the cyclotron diameter (which is the case experimentally, except for $B<10^{-2} \mathrm{~T}$ ). Classically, these edge states correspond to electrons skipping along the boundary. ${ }^{2}$ The four-terminal resistance is defined as $R_{4 t}=\left(\mu_{L}\right.$ $\left.-\mu_{R}\right) / e I$, where $\mu_{L}$ and $\mu_{R}$ are the electrochemical potentials measured by the two voltage probes shown in Fig. 2 , at the left- and right-hand side of the constriction. The left voltage probe, which is in equilibrium with the incoming electrons at the upper boundary, has $\mu_{L}=E_{F}+\Delta \mu$. We assume that the transmitted electrons in the edge states near the right voltage probe have reached a local equilibrium. This seems reasonable for a voltage probe several inelastic scattering lengths away from the constriction. As discussed above, the total current $I$ is then shared equally among the $N_{\text {wide }}$ subbands in the wide regions. More preciscly, one has ${ }^{7} I=(2 e / h)$ $\times\left(\mu_{R}-E_{F}\right) N_{\text {wide, }}$, where $\mu_{R}$ is the local electrochemical potential measured by the right voltage probe. The number $N_{\text {wide }}=k_{F} l_{c} / 2$ is the number of occupied (spin degen-

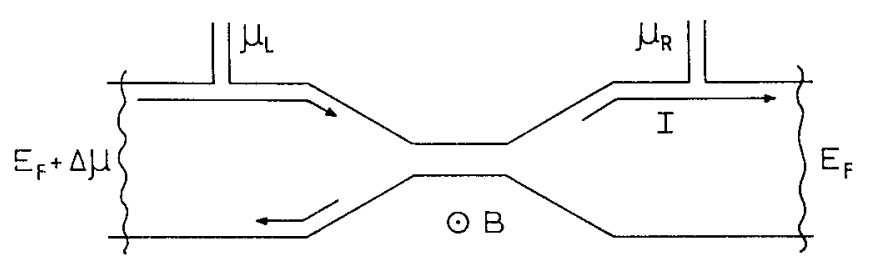

FIG. 2. Right- and left-going edge states in the ballistic regime in the presence of a magnetic field. erate) Landau levels in the wide regions. Collecting results, we find the simple formula

$$
R_{4 t}=\left(h / 2 e^{2}\right)\left(N_{\min }^{-1}-N_{\text {wide }}^{-}\right) .
$$

For a constricted electron gas with a homogeneous density, Eq. (4) predicts a negative magnetoresistance, with $R_{4 t}$ decreasing from its zero-field value $R_{2 t}$ to zero in a field range of $2 \hbar k_{F} / e W$. Physically, this is due to the fact that, as $B$ is increased, a larger and larger fraction of the edge states is transmitted through the constriction. We note that the above argument predicts a Hall resistance $R_{H}=\left(h / 2 e^{2}\right) N_{\text {wide }}^{-}$in the wide regions, unaffected by the presence of the constriction. If this result is combined with Eq. (2), one again obtains the result (4), since $R_{4 t}=R_{2 t}-R_{H}$.

We now return to a discussion of the experimental data. We note that a background resistance $R_{0}$ (about $300 \Omega$ ) is measured in series with the purely ballistic contact resistance. For gate voltages around the depletion threshold the electron-gas density is fairly uniform. In this case, we find good agreement with Eq. (4) with $W$ treated as a free parameter. This is illustrated by the data for $V_{g}=-0.3 \mathrm{~V}$ in Fig. 1. Also, the value found for the width, $0.8 \mu \mathrm{m}$, is quite plausible, the lithographic width of the split in the gate being $\sim 1 \mu \mathrm{m}$.

The magnetoresistance at higher negative gate voltages is somewhat more complicated, because of the reduction of the carrier density $n_{s}$ in the constriction. ${ }^{9}$ The result (4) for the four-terminal resistance now contains a reduced value for $k_{F}=\left(2 \pi n_{s}\right)^{1 / 2}$ in the expression for $N_{\min }$. This explains the crossover to a positive magnetoresistance observed at higher gate voltages (with nonzero Shubnikov-de Haas minima at high fields). In Fig. 1, we have only presented data up to $B=0.6 \mathrm{~T}$, because for higher fields the Hall resistance measured over the wide regions shows deviations from the value $\left(h / 2 e^{2}\right)\left(k_{F} l_{c} /\right.$ 2) ${ }^{-1}$ which would follow from the average density in the wide regions. (These deviations may be due to inhomogeneities in the carrier density, leading to filamentary current flow in this high-field regime.) The two-terminal resistance in the quantum Hall regime is only determined by the carrier density in the constriction. We can thus independently find this density from the high-field twoterminal resistance. The weak-field magnetoresistance is subsequently fitted to Eq. (4), with a constant background resistance $R_{0}=315 \Omega$ for the curves corresponding to $V_{g}=-1$ to $-3 \mathrm{~V}$. In view of the fact that the constriction width $W$ is the only free parameter, we consider the agreement found in Fig. 1 for a range of gate voltages to be quite satisfactory. Also, the resulting parameter values are reasonable.

The negative magnetoresistance effect described in this paper has a different physical origin than the effect found by Choi et al. ${ }^{10}$ in narrow multiprobe channels with a probe separation $L$ much longer than the mean free path $l$. We discuss this effect for the constriction geometry relevant to this paper (for $L \gg l$ these geometries should be equivalent). The relation between the two effects can be understood from the Landauer ${ }^{7}$ formula $R_{2 t}=(h /$ $\left.2 e^{2}\right) N_{\min }^{-1}(1-r)^{-1}$. This formula is the extension of Eq. (2) to the case that a nonzero fraction $r$ of the electrons 
injected into the constriction scatters back into the wide $2 \mathrm{D}$ EG regions. ${ }^{11}$ The corresponding expression for the four-terminal resistance is $R_{4 t}=\left(h / 2 e^{2}\right)$ $\times\left[N_{\min }^{-1}(1-r)^{-1}-N_{\text {wide }}^{-1}\right]$. As one can see from Eq. (3), the number of occupied subbands $N_{\min }$ in the constriction is approximately field independent for $2 l_{c} \gtrsim W$. The magnetoresistance effect discussed earlier (for $r=0$ ) is thus mainly due to the field dependence of $N_{\text {wide. }}$. We now argue that the effect of Choi $e t$ al. ${ }^{10}$ is caused by a reduction of the backscattering probability $r$ in this field range. The reason is that, upon raising the field, the left- and rightmoving electrons in the constriction become increasingly localized in edge states at opposite boundaries, ${ }^{7}$ so that backscattering (which requires scattering from one boundary to the other) is less likely to occur. Finally, we remark that one can see an effect closely related to the effect found by Choi et al. in the magnetoresistance data of multiprobe ballistic channels, reported in Ref. 1. In these devices, a nonzero $r$ is likely to occur because the voltage probes on the side of the channel act as diffuse scatterers. The reduction of $r$ in a weak magnetic field then again leads to a negative magnetoresistance. ${ }^{12}$ It would be of interest to study the field dependence of the backscattering probability $r$ theoretically.

The authors thank M. E. I. Broekaart for experimental assistance, and Y. Imry, B. J. van Wees, and J. G. Williamson for valuable discussions.
${ }^{1}$ G. Timp, A. M. Chang, P. Mankiewich, R. Behringer, J. E. Cunningham, T. Y. Chang, and R. E. Howard, Phys. Rev. Lett. 59, 732 (1987); M. L. Roukes, A. Scherer, S. J. Allen, Jr., H. G. Craighead, R. M. Ruthen, E. D. Beebe, and J. P. Harbison, ibid. 59, 3011 (1987).

${ }^{2}$ H. van Houten, B. J. van Wees, J. E. Mooij, C. W. J. Beenakker, J. G. Williamson, and C. T. Foxon, Europhys. Lett. (to be published).

${ }^{3}$ B. J. van Wees, H. van Houten, C. W. J. Beenakker, J. G. Williamson, L. P. Kouwenhoven, D. van der Marel, and C. T. Foxon, Phys. Rev. Lett. 60, 848 (1988).

${ }^{4}$ D. A. Wharam, T. J. Thornton, R. Newbury, M. Pepper, H. Ahmed, J. E. F. Frost, D. G. Hasko, D. C. Peacock, D. A. Ritchie, and G. A. C. Jones, J. Phys. C 21, L209 (1988).

${ }^{5}$ See also, J. Kirtley, Z. Schlesinger, T. N. Theis, F. P. Milliken, S. L. Wright, and L. F. Palmateer, Phys. Rev. B 34, 1384 (1986).

${ }^{6}$ T. J. Thornton, M. Pepper, H. Ahmed, D. Andrews, and G. J. Davies, Phys. Rev. Lett. 56, 1198 (1986).

${ }^{7}$ R. Landauer, IBM J. Res. Dev. 1, 223 (1957); R. Landauer, Z. Phys. B 68, 217 (1987); M. Büttiker, Phys. Rev. B 33, 3020 (1986); P. Streda, J. Kucera, and A. H. MacDonald, Phys. Rev. Lett. 59, 1973 (1987).
${ }^{8}$ B. J. van Wees, L. P. Kouwenhoven, H. van Houten, C. W. J. Beenakker, J. E. Mooij, J. J. Harris, and C. T. Foxon (unpublished).

${ }^{9}$ The reduced electron-gas density for high gate voltages is confirmed by measurements of the Hall voltage over the narrow channel (employing two additional gates and probes, not drawn in the inset of Fig. 1).

${ }^{10}$ K. K. Choi, D. C. Tsui, and S. C. Palmateer, Phys. Rev. B 33, 8216 (1986); see also, H. van Houten, C. W. J. Beenakker, M. E. I. Broekaart, M. G. J. Heijman, B. J. van Wees, J. E. Mooij, and J. P. André, Acta Electron. (to be published).

"If one treats the constriction resistance $\left(h / 2 e^{2}\right)\left(\pi / k_{F} W\right)$ and the Drude resistance $\left(h / e^{2}\right)\left(k_{F} l_{e}\right)^{-1}(L / W)$ in the absence of a magnetic field additively, it follows that $r$ is simply given by $(1-r)^{-1}=1+\left(2 L / \pi l_{e}\right)$. Under the conditions of our experiment, we would then estimate $r \sim 0.2$, so that a small fraction of the observed magnetoresistance may actually be due to the effect of Ref. 10.

${ }^{12}$ The anomalies around zero magnetic field observed in the narrowest channels in Ref. 1 are not explained by this classical argument. A quantum-mechanical explanation for the quenching of the Hall effect in narrow channels is proposed in C. W. J. Beenakker and H. van Houten (unpublished). 